\title{
Pearls \& Oy-sters: The critical role of histopathology in diagnosing cancer-associated necrotizing CNS vasculitis
}

Joshua Sheehan, MD, Jessica Tate, MD, Ryan Mott, MD, Carol Geer, MD, Rachel Wolfe, MD, Roy E. Strowd, MD, and Amy Guzik, MD

Neurology ${ }^{\circledR}$ 2018;90:808-811. doi:10.1212/WNL.0000000000005350

\section{Abstract}

\section{Objective}

To highlight the importance of a broad differential and histopathologic confirmation in patients with newly diagnosed cancer with brain lesions atypical for CNS metastasis.

\section{Methods}

We report 2 cases of biopsy-proven CNS vasculitis in patients undergoing treatment for a newly diagnosed nonmetastatic cancer. Comprehensive medical record review was performed to identify the clinical presentation, representative neuroimaging, histopathologic features, and response to treatment.

\section{Results}

Patient 1 presented 1 month into induction therapy of malignant vaginal squamous cell carcinoma (stage 3, T2N1M0) with acute episodic left-sided hemiparesis due to seizure activity progressing to severe encephalopathy. Imaging revealed a right frontoparietal lesion while systemic workup was unrevealing. Biopsy demonstrated necrotizing vasculitis. Patient 2 presented 6 months after diagnosis of right breast invasive ductal carcinoma (stage IIa, T2N0M0, estrogen receptor-positive, progesterone receptor-positive, human epidermal growth factor receptor-2 positive) with subacute bifrontal headaches with associated phonophobia. Imaging showed hyperintense lesions involving the right temporoparietal region and systemic workup was unrevealing. Brain biopsy showed a necrotizing vasculitis. Patient 1 was treated with methyprednisolone and plasmapheresis and patient 2 was treated with prednisone. Both patients showed complete resolution of symptoms shortly after treatment and improvement on imaging.

\section{Conclusions}

These cases highlight the importance of comprehensive evaluation of new brain lesions in patients with nonmetastatic solid tumors. Characteristics of new brain lesions in patients with cancer that should raise suspicion of diagnoses other than brain metastasis include (1) primary malignancy without regional or distant metastasis, (2) imaging without discrete mass-like enhancement, and (3) cortically based location of lesions not at the gray-white matter junction.

\author{
Correspondence \\ Dr. Strowd \\ rstrowd@wakehealth.edu
}




\section{Glossary}

AChR = acetylcholinesterase receptor; ANA = antinuclear antibody; PCNSV = primary CNS vasculitis; SCNSV = secondary CNS vasculitis.

\section{Pearls}

- Cancer-associated CNS vasculitis is a rare but important cause of new brain lesion in a patient with systemic malignancy.

- Characteristics of new brain lesions in cancer patients that should raise suspicion of diagnoses other than brain metastasis include (1) primary malignancy without regional or distant metastasis, (2) imaging without discrete masslike enhancement, and (3) cortically based location of lesions not at the gray-white matter junction.

- Histopathologic confirmation is important in patients with newly diagnosed cancer with CNS lesions atypical for metastatic disease.

\section{Oy-ster}

- Radiographic findings of CNS vasculitis are nonspecific and can mimic neoplasm or other pathology.

Brain lesions are present on MRI in up to $40 \%$ of patients with cancer. ${ }^{1}$ While the majority represent metastases, up to $11 \%$ are radiographic mimics that are difficult to distinguish by imaging alone. ${ }^{1}$ Metastatic disease typically appears as sharply demarcated solid or ring-enhancing lesions with surrounding edema. ${ }^{1}$ These imaging features, however, overlap with less common etiologies including inflammatory, vascular, and infectious pathology. Histology remains the gold standard for diagnosis and is critically important in atypical cases. ${ }^{2}$

We report 2 patients with early stage nonmetastatic systemic cancer who developed new neurologic symptoms and cortically based brain lesions during first-line therapy.

\section{Patient 1}

A 70-year-old woman was diagnosed with malignant vaginal squamous cell carcinoma (stage 3, T2N1M0) in March 2016. Induction therapy consisted of radiation (45 Gy) and cisplatin $\left(40 \mathrm{mg} / \mathrm{m}^{2}\right.$ weekly). One month into treatment (fraction $17 / 25$ and cycle 3), she presented with acute onset of episodic left hemibody weakness. CT of the head showed no acute hemorrhage or early ischemic findings. Once admitted, she developed intermittent uncontrolled rhythmic left upper extremity jerking concerning for seizure.

Continuous video EEG monitoring demonstrated an epileptogenic focus in the right central region and levetiracetam was started. Brain MRI without contrast showed a linear, cortically based right frontoparietal lesion of unclear etiology (figure 1A). Repeat gadolinium-enhanced MRI (figure 1A) showed mass-like progression of cortical gyra swelling and right precentral and postcentral gyral edema without enhancement. By day 8 , imaging revealed increased swelling in the gyri of the posterior right frontal and anterior right parietal lobe with surrounding edema, hemorrhage, and local mass effect (figure 1A). CT angiography of the head was not suggestive for vasculopathy or aneurysm. Clinically, the patient continued to demonstrate intermittent left-sided weakness. Differential diagnosis included infectious encephalitis, simple partial status epilepticus with secondary radiographic abnormalities, vasculitis, or chemotherapeutic toxicity. Brain metastasis was considered given the mass-like expansion (figure 1A) but was considered less likely due to the lack of contrast enhancement.

Lumbar puncture showed an inflammatory profile with no evidence of malignancy on cytology (table e-1, links.lww. $\mathrm{com} / \mathrm{WNL} / \mathrm{A} 382$ ). Systemic workup showed no evidence of systemic infection or vasculitis. Autoimmune/paraneoplastic testing revealed no classic antibody-associated encephalitides or vasculitides except for an elevated acetylcholinesterase receptor $(\mathrm{AChR})$ binding antibody $(0.11 \mathrm{nmol} / \mathrm{L})$.

Given the lack of definitive diagnosis with noninvasive testing, brain biopsy was performed showing a nongranulomatous necrotizing vasculitis characterized by fibrinoid necrosis of leptomeningeal blood vessels and brain tissue gliosis (figure e-1, links.lww.com/WNL/A381). There was no evidence of systemic vasculitis and the patient was diagnosed with isolated CNS vasculitis. Five days of IV methylprednisolone (1,000 mg daily) was administered without clinical improvement. Cyclophosphamide and rituximab were deferred in context of recent chemotherapy and instead the patient underwent 7 sessions of plasmapheresis with substantial cognitive and motor improvement. Brain MRI performed 2 weeks following treatment showed improvement and she has remained stable on a prednisone $(20 \mathrm{mg})$ taper for 6 months.

\section{Patient 2}

A 67-year-old woman was diagnosed with right breast invasive ductal carcinoma (stage IIa, T2NOM0, estrogen receptorpositive, progesterone receptor-positive, human epidermal growth factor receptor-2 positive) and subsequently underwent a lumpectomy. She presented with subacute worsening of bifrontal headaches waking her from sleep. Headaches had increased in frequency and severity over 6 months with associated phonophobia and lightheadedness but no aura, vision changes, or other focal symptoms. Despite prophylactic and abortive medications, she experienced minimal relief. 


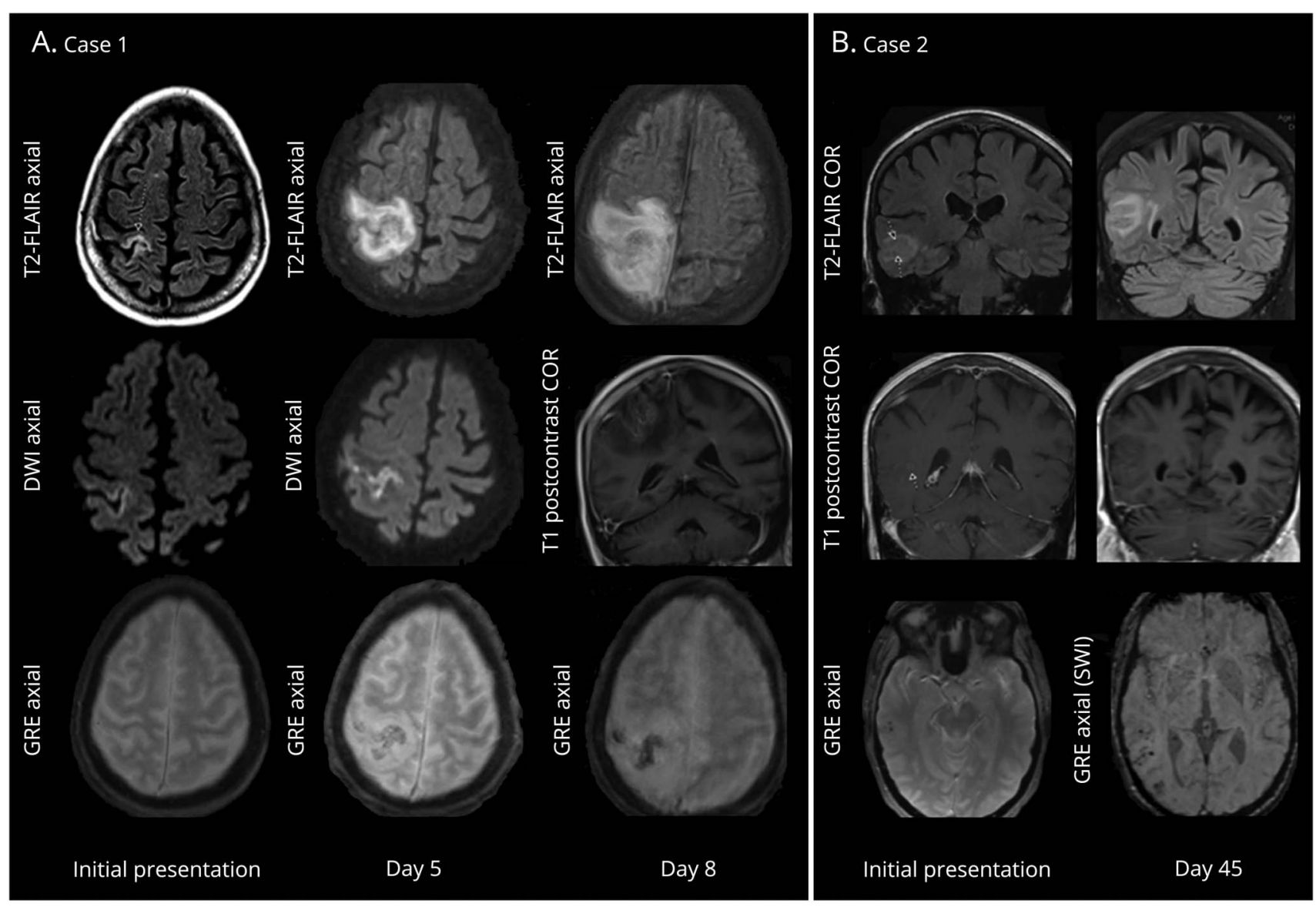

(A) Case 1: top row: progression of high T2/fluid-attenuated inversion recovery (FLAIR) signal/edema in the right frontoparietal lobe; middle row: mild right frontoparietal cortically based restricted diffusion, faint right frontoparietal gyral enhancement; bottom row: progressive susceptibility artifact (hemorrhage) on gradient imaging in the region of signal abnormality. (B) Case 2: top row: progressive high T2/FLAIR signal/edema in right temporal and parietal lobes; middle row: very faint leptomeningeal enhancement in region of edema; bottom row: progressive cortically based susceptibility artifact (hemorrhage) in region of edema.

MRI brain was ordered for further evaluation and demonstrated a T2-weighted hyperintense mass lesion involving the right temporoparietal cortex with local swelling, subtle leptomeningeal enhancement, and no restricted diffusion on diffusion-weighted imaging (figure 1B). Initial concerns included bran metastasis vs low-grade glioma. Brain biopsy was recommended and revealed a nongranulomatous necrotizing vasculitis with small leptomeningeal blood vessels with destructive changes (figure e-1, links.lww.com/WNL/A381).

Lumbar puncture was negative for malignancy but showed polyclonal lymphocytosis (table e-1, links.lww.com/WNL/ A382). Paraneoplastic and autoimmune testing was negative except for a weakly positive antinuclear antibody (ANA) (1:40 speckled). There was no evidence of systemic vasculitis and the patient was diagnosed with isolated CNS vasculitis. She was started on an outpatient regimen of prednisone $60 \mathrm{mg} / \mathrm{d}$ with no evidence of recurrent vasculitis at 12 months.

\section{Discussion}

Brain metastases may occur in up to $40 \%$ of patients with cancer. ${ }^{1}$ The 2 cases presented here underscore the importance of a differential diagnosis in evaluating atypical cases of new mass-like brain lesions in patients with cancer. Brain metastasis was considered in both patients; however, clinical and imaging findings were atypical for CNS metastasis, including early stage cancer without known systemic metastasis, cortically based brain lesions, and atypical patterns of gadolinium enhancement. In these cases, a broad differential diagnosis was important and ultimately histopathology was critical in confirming the rare diagnosis of CNS vasculitis.

CNS vasculitis is a rare form of vasculitis involving the cerebral vasculature. It is broadly categorized into primary CNS vasculitis (PCNSV; also referred to as primary angiitis of the CNS) and secondary CNS vasculitis (SCNSV) based on the presence of systemic manifestations of vasculitis. While SCNSV has been attributed to a wide array of systemic 
vasculitides and inflammatory diseases, the causes of PCNSV are largely unknown. CNS vasculitis has been rarely associated with systemic malignancy. Case reports of CNS vasculitis have been associated with Hodgkin disease, ${ }^{3}$ leukemia, ${ }^{4}$ and metastatic breast cancer. ${ }^{5}$ Similar to these prior reports, our patients presented subacutely with insidious headache or focal neurologic deficit.

Radiographic findings were atypical for metastasis. Imaging showed cortically based lesions that lacked the typical enhancing pattern seen in metastasis. In case 1, the linear pattern of restricted diffusion concentrated in the right frontoparietal cortex was initially suspicious for encephalitis. In case 2 , glioma was considered given the discrete mass-like nature of the lesion with surrounding edema. This lesion did not demonstrate the typical contrast enhancement of a breast metastasis and the atypical location, faint leptomeningeal enhancement, and a susceptible artifact on gradient echo sequences did not fit with the classic pattern of a primary glioma. In both of these cases, imaging characteristics were insufficient to establish a diagnosis and further investigation with biopsy was warranted.

In these 2 cases, a histopathologic diagnosis of necrotizing vasculitis was unexpected. As in these cases, ambiguous clinical presentation and radiographic imaging present diagnostic and therapeutic predicaments, and providers must weigh the benefits of early treatment with the risks of an invasive confirmatory biopsy. Given the risks of surgery and advancements of noninvasive treatments including stereotactic radiosurgery for managing cerebral metastasis in metastatic cancer, clinicians may consider therapeutic intervention without histopathologic confirmation. ${ }^{6}$ However, in both of these cases the diagnosis of CNS vasculitis and subsequent choice of immunosuppressive treatment would not have been identified without histopathologic evaluation.

Current treatment strategies for CNS vasculitis typically include high-dose glucocorticoids with or without concurrent cyclophosphamide treatment, with rituximab or azathioprine reserved as second-line treatment. ${ }^{7}$ In a retrospective review, Salvarani et al. ${ }^{8}$ found a favorable response to treatment in $81 \%$ of patients, highlighting the treatable nature of this condition and need for early accurate diagnosis. Treatment of CNS vasculitis in patients with a systemic malignancy undergoing chemoradiotherapy has not yet been defined. Our first patient experienced clinical response to glucocorticoids followed by plasmapheresis. The importance of concomitantly treating the underlying cancer in these patients is unknown; further research is warranted.

The etiopathogenesis of CNS vasculitis in patients with systemic malignancy has not been determined. Recent literature suggests cerebral amyloid deposits may trigger CNS vasculitis, but the exact mechanism remains speculative. ${ }^{9}$ Interestingly, our first patient developed PCNSV soon after initiation of radiation therapy. Radiation therapy has been shown to affect immunologic activation, ${ }^{4}$ and the abscopal effect has been used to describe distant response of metastatic lesions following local irradiation. ${ }^{10}$
It has also been implicated in the formation of atherosclerosis and cardiovascular complications ${ }^{11}$ through the systemic activation of cytokines and growth factors (such as tumor growth factor- $\beta 1$ and interleukin-1 $\beta) .{ }^{11}$ It is possible that a similar type of immune modulation occurred in this patient that ultimately manifested in CNS vasculitis, although this is difficult to assess.

The interplay between cancer and the immune system is complex. In the last decade, awareness of paraneoplastic neurologic syndromes has broadened with the description of increasing numbers of specific cancer-associated antibodies. Whether cancer-associated CNS vasculitis represents a paraneoplastic neurologic syndrome is not certain. Nonspecific circulating antibodies (AChR, ANA) were present in both cases without clear association to pathology.

These cases highlight the importance of a broad differential, evaluating mimics, and pursuing a histopathologic diagnosis for cancer patients presenting with a new brain lesion with ambiguous radiographic and clinical findings.

\section{Author contributions}

Joshua Sheehan (medical student): lead role in writing manuscript. Roy E. Strowd (faculty mentor): acquisition of data, critical revision of manuscript for intellectual content. Amy Guzik (faculty mentor): acquisition of data, critical revision of manuscript for intellectual content. Jessica Tate: critical revisions, intellectual contribution. Rachel Wolfe: critical revisions, intellectual contribution. Carol Geer: critical revisions, intellectual contribution, figure design. Ryan Mott: acquisition of data, figure design.

\section{Study funding}

No targeted funding reported.

\section{Disclosure}

The authors report no disclosures relevant to the manuscript. Go to Neurology.org/N for full disclosures.

\section{References}

1. Campos S, Davey P, Hird A, Pressnail B, Bilbao J, Aviv RI. Brain metastasis from an unknown primary, or primary brain tumour? A diagnostic dilemma. Curr Oncol 2009;16:62-66.

2. Parisi JE, Moore PM. The role of biopsy in vasculitis of the central nervous system. Semin Neurol 1994;14:341-348.

3. Rosen CL, Depalma L, Morita A. Primary angiitis of the central nervous system as a first presentation in Hodgkin's disease: a case report and review of the literature. Neurosurgery 2000;46:1504.

4. Hasler P, Kistler H, Gerber H. Vasculitides in hairy cell leukemia. Semin Arthritis Rheum 1995;25:134-142.

5. Taccone FS, Salmon I, Marechal R, Blecic SA. Paraneoplastic vasculitis of central nervous system presenting as recurrent cryptogenic stroke. Int J Clin Oncol 2007;12:155-159.

6. Mintz A, Perry J, Spithoff K, Chambers A, Laperriere N. Management of single brain metastasis: a practice guideline. Curr Oncol 2007;14:131-143.

7. Joseph FG, Scolding NJ. Cerebral vasculitis: a practical approach. Pract Neurol 2002; 2:80-93.

8. Salvarani C, Brown RD Jr, Calamia KT, et al. Primary central nervous system vasculitis: analysis of 101 patients. Ann Neurol 2007;62:442-451.

9. Fountain NB, Eberhard DA. Primary angiitis of the central nervous system associated with cerebral amyloid angiopathy: report of two cases and review of the literature. Neurology 1996;46:190-197.

10. Lock M, Muinuddin A, Kocha WI, et al. Abscopal effects: case report and emerging opportunities. Cureus 2015;7:e344.

11. Basavaraju SR, Easterly CE. Pathophysiological effects of radiation on atherosclerosis development and progression, and the incidence of cardiovascular complications. Med Phys 2002;29:2391-2403. 


\section{Neurology}

\section{Pearls \& Oy-sters: The critical role of histopathology in diagnosing cancer-associated necrotizing CNS vasculitis}

Joshua Sheehan, Jessica Tate, Ryan Mott, et al.

Neurology 2018;90;808-811

DOI 10.1212/WNL.0000000000005350

\section{This information is current as of April 23, 2018}

\section{Updated Information \& Services}

References

Subspecialty Collections

\section{Permissions \& Licensing}

Reprints including high resolution figures, can be found at: http://n.neurology.org/content/90/17/808.full

This article cites 11 articles, 1 of which you can access for free at: http://n.neurology.org/content/90/17/808.full\#ref-list-1

This article, along with others on similar topics, appears in the following collection(s):

All Clinical Neurology

http://n.neurology.org/cgi/collection/all_clinical_neurology All Education

http://n.neurology.org/cgi/collection/all_education

All Imaging

http://n.neurology.org/cgi/collection/all_imaging

Metastatic tumor

http://n.neurology.org/cgi/collection/metastatic_tumor

Vasculitis

http://n.neurology.org/cgi/collection/vasculitis

Information about reproducing this article in parts (figures,tables) or in its entirety can be found online at:

http://www.neurology.org/about/about_the_journal\#permissions

Information about ordering reprints can be found online:

http://n.neurology.org/subscribers/advertise

Neurology ${ }^{\circledR}$ is the official journal of the American Academy of Neurology. Published continuously since 1951, it is now a weekly with 48 issues per year. Copyright () 2018 American Academy of Neurology. All rights reserved. Print ISSN: 0028-3878. Online ISSN: 1526-632X.

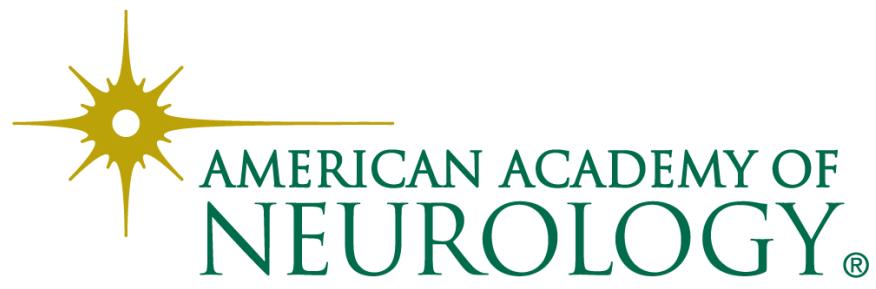

